\title{
Niveau d'énonciation, niveaux de culture en anglais informatique
}

L'incidence de la culture professionnelle de l'apprenant sur la nature de la langue de spécialité à lui enseigner

\section{Anne Pradeilles}

\section{(2) OpenEdition}

\section{Journals}

Édition électronique

URL : http://journals.openedition.org/asp/4494

DOI : $10.4000 /$ asp.4494

ISBN : 978-2-8218-0396-1

ISSN : 2108-6354

\section{Éditeur}

Groupe d'étude et de recherche en anglais de spécialité

\section{Édition imprimée}

Date de publication : 1 mars 2004

Pagination : 11-34

ISSN : 1246-8185

\section{Référence électronique}

Anne Pradeilles, « Niveau d'énonciation, niveaux de culture en anglais informatique », ASp [En ligne], 43-44 | 2004, mis en ligne le 11 juin 2014, consulté le 19 avril 2019. URL : http://

journals.openedition.org/asp/4494; DOI : 10.4000/asp.4494

Ce document a été généré automatiquement le 19 avril 2019

Tous droits réservés 


\section{Niveau d'énonciation, niveaux de culture en anglais informatique}

L'incidence de la culture professionnelle de l'apprenant sur la nature de la langue de spécialité à lui enseigner

\section{Anne Pradeilles}

1 Dans cet énoncé, les deux grandes variables ou paramètres sont les mots clés suivants : qualification professionnelle et compétence linguistique. Ils constituent la base de cette étude qui a pour objectif de démontrer l'incidence de ces deux paramètres sur le type d'enseignement de spécialité à dispenser. Ainsi l'enseignant doit bien maîtriser les deux axes et être capable de situer l'apprenant sur une grille à double entrée, à partir de laquelle il doit déduire les besoins de l'apprenant et la nature de l'enseignement à lui dispenser.

2 Je situe donc cette étude dans une optique de pédagogie centrée sur l'apprenant et je base l'enseignement à dispenser sur une analyse des besoins linguistiques et professionnels. Ayant constaté que ces deux grands paramètres, professionnel et linguistique, ont une incidence sur la nature de l'enseignement à dispenser, j'essaierai de formuler quelques hypothèses théoriques.

3 J'appuie ma démonstration sur un certain nombre de travaux antérieurs (cf. bibliographie). J'adopte les propositions théoriques de Janine Gallais-Hamonno pour la langue de spécialité; je fais référence à Jean Tournier pour la lexicologie, à Peter Roe pour son approche par tâches à accomplir, à Louis Trimble pour son analyse du discours scientifique, et j'utilise les études de Pauline Robinson et de Jo Mc Donough sur la langue de spécialité en général. Après les énoncés théoriques, j’étudierai des pédagogies de la langue scientifique, en utilisant leurs longs sous-titres, et j'en tirerai plusieurs exemples.

4 Je voudrais aussi rappeler que cette étude est une sorte de synthèse de mes propres travaux en anglais informatique sur les aspects suivants : les différents niveaux de langue pour un même fait scientifique ; les quatre niveaux d'un énoncé ; les articles the, $a / a n, \varnothing$; les formes verbales, le résumé en anglais de spécialité ; la constitution d'un dictionnaire d'anglais des réseaux avec des étudiants; l'interdisciplinarité et la pluridisciplinarité. 
Pour toutes ces recherches, qui ont fait l'objet de communications et d'articles, j'ai étudié de nombreux textes d'anglais informatique et je ne ferai ici référence qu'à quelques exemples extrêmes.

5 Enfin, cette étude est aussi destinée à constituer une étape sur la voie de notre recherche collective au sein du DEA d'anglais de spécialité créé à la rentrée universitaire 1991-1992 à l'université de Bordeaux 2, Toulouse 1 et Montpellier 3. Elle a pour but de rappeler l'existence de quelques précurseurs à connaître et d'apporter des pistes de réflexion.

6 La bibliographie a donc une importance particulière ainsi que les croquis et diagrammes qui représentent, souvent mieux qu'un long discours, les «théorèmes " que j'utilise ou que j'avance personnellement. Mon exposé sera divisé en trois parties: un bref bref panorama de l'analyse de la langue de spécialité dans lequel je me place à partir de l'hypothèse théorique que J. Gallais-Hamonno énonce dans son article «Niveaux d'énonciation, niveaux de culture » (qui a fourni le titre et le point de départ de ce travail) et en m'appuyant sur mon expérience personnelle de seize ans d'enseignement de l'anglais informatique, je proposerai un certain nombre de croquis et schémas représentant "l'incidence du niveau de culture professionnelle de l'apprenant sur la nature de la langue de spécialité à lui enseigner ", intitulé de mon sous-titre. Dans une troisième partie, je montrerai par quelques exemples concrets de documents authentiques d'anglais informatique combien il est nécessaire pour l'enseignant de langue de spécialité de mieux maitriser les deux axes «langue et spécialité », pour luimême et pour son apprenant.

7 J'utiliserai les travaux de pédagogues de l'anglais scientifique destinés à des scientifiques de langue anglaise pour bien montrer comment ce type de texte authentique peut être utilisé au mieux comme document pédagogique d'apprentissage de la langue. Je montrerai combien un texte hyperspécialisé est plus facile à comprendre pour nos étudiants qu'un texte de vulgarisation ou de pédagogie "amélioré » par l'humour. Je citerai enfin quelques extraits de copies d'étudiants pour montrer que mes hypothèses théoriques sont ancrées dans une pratique pédagogique de tous les jours.

\section{Bref panorama de l'anglais de spécialité}

\subsection{Rappel des définitions}

8 Pauline Robinson consacre le premier chapitre de son livre ESP à ces efforts de définitions (1980: 5-14). Plus que des mots, nous en retiendrons d'excellents croquis (cf. annexe 1).

\subsection{Les acteurs du processus de communication}

9 Du remarquable English for Science and Technology: A Discourse Approach de Louis Trimble (1985), nous retiendrons ici le chapitre 4 , « The individualizing process » :

Individualization of assignments (that is, the individualization process) is defined as the process of finding a way to create a set of general assignments that can be used by each of the students in a given class in respect to their individual needs. This type of individualizing makes a meaningful connection between what the students have learned in their English class and what they have learned (or already know about) in their subject-matter class and/or their fields of interest. (1985: 23) 
Dans ESP in Perspective: A Practical Guide, Jo Mc Donough souligne « the many faces of ESP ». Dans son chapitre 2, elle étudie les «fundamental variables » de la relation pédagogique spécifique en langue de spécialité, certaines variables sont "community-controlled», d'autres «teacher controlled», d'autres enfin sont des «learner variables». L'enseignant doit donc s'adapter et devenir comme 1'écrit Strevens, « a polymath teacher ", " chameleonlike ». Dans son chapitre 3, «Analysis of learners' needs », elle analyse les «needs » par rapport aux «wants» $(1984: 36)$ et estime que les deux doivent être reliés en une « integrated procedure $»(1984: 40)$.

\subsection{Les moyens de situer l'apprenant sur une grille d'évaluation}

11 Nous adopterons ici les trois grilles proposées par Peter Roe dans «A task-based approach to ESP » : niveau linguistique général ; échelle des performances par rapport aux tâches linguistiques et échelle des performances par rapport aux tâches données dans les activités scientifiques et le contexte professionnel (Roe 1991 : 108-110).

\subsection{Terminologie et lexique}

12 D'un point de vue théorique général, nous ferons référence au Précis de lexicologie anglaise de Jean Tournier. L'exemple de la « constellation lexicale » du mot « sense » (1988:44) est tout à fait intéressant et adaptable à l'anglais de spécialité. Le radical «COMPUT- » en anglais informatique par exemple, pris dans le Webster's Dictionary, offre un remarquable échantillon d'affixes, suffixes et préfixes permettant da former des noms, verbes, adjectifs et adverbes.

Patrick Hanks dans son article «A New kind of Dictionary for English Learners: Cobuild » (1988) et Michèle Rivas, « Dictionnaire anglais unilingue et autonomie de l'apprentissage, de l'utilité du Collins Cobuild English Language Dictionary en milieu universitaire » (1988) soulignent bien l'importance des dictionnaires et lexiques comme outils d'enseignement et d'apprentissage en langue de spécialité. L'APLIUT a consacré à ce thème son $\mathrm{XI}^{\mathrm{e}}$ congrès (Les Cahiers de l'APLIUT 1990).

Les travaux du GEPE sur le français en informatique et la pidginisation du français par le jargon américain de l'informatique font clairement ressortir le rôle pr6pondérant des professionnels dans la créativité terminologique d'une langue de ep6cialité.

15 L'approche quantitative s'est révélée fort pertinente en langue de spécialité et nous renvoyons le lecteur à plusieurs études :

- Hanks et les travaux de l'équipe COBUILD : dans la langue générale, sur dix-huit millions de tokens (termes) dans le corpus, le premier mot porteur de sens est «time», en $70^{\circ}$ position seulement. C'est très différent en langue de spécialité: le scientifique ne craint pas la répétition et la fréquence d'un terme est un marqueur de mot clé.

- Dans une étude précédente (1981c), j’ai montré combien la quantification peut guider le chercheur. Plusieurs articles de l'ILSER en fournissent aussi de bons exemples.

- Computational Linguistics, la revue de l'ALLC (Association for language and Literature Computing), créée en 1986, constitue un modèle du genre. Susan Hockey a présenté ses travaux au Xe colloque du GERAS à Toulouse en 1989. Son analyse textuelle est illustrée par des exemples de littérature générale mais peut s'appliquer à tout corpus. 
De ce très bref panorama on peut constater qu'il reste encore une étape à franchir : une synthèse des multiples travaux de recherche, qui devrait aboutir à la rédaction d'une "grammaire» de l'anglais de spécialité. Le Programme pédagogique national des départements Informatique d'IUT rédigé par le groupe informatique de l'APLIUT a fait des propositions de têtes de chapitre à la Commission pédagogique nationale. Ces propositions 1988, remises à jour en mai 1992, figurent dans Les Cahiers de l'APLIUT 28-29.Ca travail a aussi été fait pour d'autres spécialités. Il reste aujourd'hui à regrouper ces travaux, les comparer et à en faire une synthèse faisant apparaître les convergences aussi bien que les divergences entre les syntaxes de toutes ces langues de spécialité.

\section{Niveaux d'énonciation, niveaux de culture en anglais informatique : incidence du niveau de culture professionnelle de l'apprenant sur la nature de la langue de spécialité à lui enseigner}

\subsection{Les aspects théoriques}

17 L'article fondamental de J. Gallais-Hamonno, "Niveaux d'énonciation, niveaux de culture ", sera résumé ici par quatre tableaux : les niveaux d'énonciation dans un texte scientifique ; les langues différentes des communautés culturelles différentes en un temps donné de la culture scientifique; progrès scientifique et développement de la langue: niveau de culture scientifique selon la période considérée ; enrichissement progressif des langues de spécialité et de communication : à noter les niveaux 1 et 2 correspondent à la langue de spécialité et les niveaux 3 et 4 à la langue de communication. Un exemple pour résumer la démonstration théorique : "1'utilisateur d'une machine à écrire comportant un effaceur » (Gallais-Hamono 1982b : 40-42).

18 Les articles de l'auteur constituent une vulgarisation et une application des aspects théoriques (cf. bibliographie).

\subsection{Proposition de représentation du processus pour les étudiants du département d'informatique d'IUT}

19 Notre ambition est de donner à l'apprenant la culture linguistique professionnelle nécessaire à sa qualification professionnelle. Le vecteur $\mathrm{t} 1$ correspond au strict bagage linguistique de base en anglais informatique: par exemple, le lexique de base des instructions informatiques et des messages-écran, le «survival level ». Si l'étudiant est un bon apprenant et si on a le temps, alors on peut aussi combler peu à peu la partie basse du rectangle par des compétences de communication (voir figure 1). 
Figure 1

\begin{tabular}{|c|c|c|c|c|c|c|}
\hline $\begin{array}{l}\text { axe } \\
\text { professionnel }\end{array}$ & & & & & & \\
\hline \multicolumn{7}{|l|}{ chercheur } \\
\hline \multicolumn{7}{|l|}{$\mathrm{bac}+5$} \\
\hline \multicolumn{7}{|l|}{$\mathrm{bac}+2$ DUI } \\
\hline $\begin{array}{l}\text { bac \& ou } \\
\text { bac général }\end{array}$ & & $\prod_{1}<$ & $\frac{\text { ssp }}{--\operatorname{com}}$ & munication & $\prod_{\text {in }}$ & \\
\hline \multicolumn{7}{|l|}{ néophyte } \\
\hline $\begin{array}{l}\text { axe } \\
\text { linguistique }\end{array}$ & débutant & Moyen - & Moyen & Moyen + & Avance & Bilingue \\
\hline
\end{tabular}

Figure 2. Un exemple concret, les cinq semestres de formation à l'IUT

\begin{tabular}{|c|c|c|c|c|}
\hline Bac +0 & lère année $\mathrm{T2}$ & $T^{3}$ & 2ème année $T 4$ & DUT \\
\hline niveau 1 & niveau 2 & niveau 3 & niveau 4 & niveau 5 \\
\hline $\begin{array}{l}\text { Informatique } \\
0 \text { : niveau } \\
\text { utilisateur }\end{array}$ & $\begin{array}{l}\text { lers langages } \\
\text { découverte } \\
\text { des machines }\end{array}$ & $\begin{array}{l}\text { "noyau dur" } \\
\text { de la } \\
\text { discipline }\end{array}$ & $\begin{array}{l}\text { vers la } \\
\text { competence } \\
\text { professionnel. }\end{array}$ & $\begin{array}{l}\text { analyste- } \\
\text { programmeur } \\
\text { débutant }\end{array}$ \\
\hline $\begin{array}{l}\text { ANGLAIS : } \\
\text { evaluation } \\
\text { des } 4 \\
\text { "gkilis" }\end{array}$ & $\begin{array}{l}\text { Langue : pre- } \\
\text { specialitê } \\
\text { culture/civ. } \\
\text { professionnel. }\end{array}$ & $\begin{array}{l}\text { Début de } \\
\text { langue de } \\
\text { specialité }\end{array}$ & $\begin{array}{l}\text { Langue de } \\
\text { epécialité }\end{array}$ & $\begin{array}{l}\text { Compétence } \\
\text { linguistique } \\
\text { profesaionnel. }\end{array}$ \\
\hline $\begin{array}{l}\text { Renforcement } \\
\text { du lexique et } \\
\text { de la syntaxe } \\
\text { de base }\end{array}$ & $\begin{array}{l}\text { mise en place } \\
\text { des pré-requis } \\
\text { du lexique et } \\
\text { de la syntaxe } \\
\text { de } \\
\text { pré-epécialité }\end{array}$ & $\begin{array}{l}\text { Lexique et } \\
\text { syntaxe en } \\
\text { situation : } \\
\text { textes au- } \\
\text { thentiques } \\
\text { d'AS }\end{array}$ & $\begin{array}{l}\text { Consolidation } \\
\text { de syntaxe et } \\
\text { enrichissement } \\
\text { de lexique } \\
\text { d'As }\end{array}$ & $\begin{array}{l}\text { Anglais en } \\
\text { situation } \\
\text { professionnel. } \\
\text { authentique }\end{array}$ \\
\hline $\begin{array}{l}\text { G Nominal } \\
\text { G Verbal } \\
\text { "noyau dur" } \\
\text { cf. PPN }\end{array}$ & $\begin{array}{l}\text { mesures, sigles } \\
\text { abréviations, } \\
\text { quantité, } \\
\text { comparaison, } \\
\text { modalités, etc. }\end{array}$ & $\begin{array}{l}\text { constitution } \\
\text { d'un corpus } \\
\text { terminolo- } \\
\text { gique } \\
\text { spécifique }\end{array}$ & $\begin{array}{l}\text { travaux } \\
\text { guidís } \\
\text { et } \\
\text { recherches } \\
\text { personnelles }\end{array}$ & $\begin{array}{l}\text { Projets } \\
\text { individuels } \\
\text { et echanges } \\
\text { collectifs }\end{array}$ \\
\hline \multirow{4}{*}{$\begin{array}{l}\text { Role de } \\
\text { L'ENSEIGRANT }\end{array}$} & \multirow[t]{2}{*}{ BNSEIGNant } & \multirow[t]{2}{*}{ ENSeignant } & \multirow[t]{2}{*}{ Enseignant } & s. \\
\hline & & & & \multirow[t]{3}{*}{ APPRENANTS } \\
\hline & ApprenAwr & \multirow[t]{2}{*}{ ApPRENANT (s) } & \multirow{2}{*}{ APPRBNANT (S) } & \\
\hline & & & & \\
\hline
\end{tabular}

20 Sur l'axe horizontal du temps, on passe progressivement du cours «magistral » à l'apprentissage autonome et, sur l'axe vertical de l'apprentissage et de la formation, on assiste à un enrichissement du contenu linguistique par le contenu professionnel au fur et à mesure de la professionnalisation de l'apprenant.

21 Comme le montrent les différents schémas (voir Annexe 2), on peut énoncer une sorte de théorème : le niveau de compétence professionnelle de départ, puis à un temps $t$, influe directement sur le contenu d'anglais de spécialité à acquérir. Ainsi

22 SI compétence professionnelle de haut niveau

ALORS pas de problème de LEXIQUE spécifique (souvent d'origine anglo-saxonne en anglais informatique) 
Mais peut-être des problèmes de SYNTAXE générale et donc de compréhension et d'expression écrite et orale

SI compétence linguistique de haut niveau

- ET compétence professionnelle nulle ou faible

ALORS gros travail de LEXIQUE spécifique

- ET compétence professionnelle de haut niveau

ALORS aucun problème.

23 Ainsi tous les parcours sont individuels en fonction de ces deux variables (cf. McDonough 1984 ; Trimble 1985).

\section{Quelques exemples pour illustrer ces affirmations théoriques et les étayer}

24 Je laisserai de côté le problème nouveau de «l'anglais international », omniprésent en informatique où l'anglais est la langue véhiculaire internationale. Il génère des problèmes spécifiques de compréhension tant à l'écrit, avec une certaine "pidginisation » de la syntaxe, qu'à l'oral, avec des problèmes de prononciation d'une langue souvent mal oralisée. Je ne citerai qu'un exemple, l'anglais japonais. Le rapport Jacudi, dont un extrait a été retenu dans Leray et alii (1980), présente une utilisation défectueuse des articles the, $a / a n$ et $\varnothing$, et la prononciation nippone de l'anglais dans des conférences d'informatique contraint la plupart des auditeurs à recourir à la traduction simultanée. Néanmoins, ces informaticiens se comprennent entre eux dans cet « anglais ».

Une autre atteinte à la communication est portée par le jargon informatique, le computerese. Les informaticiens se délectent de cette langue codée. Elle exclut le noninitié et constitue pour celui qui en connait les secrets un instrument de pouvoir. L'analyse des comportements sur le terrain montre que la quantité de termes jargonnants, souvent importée directement de l'anglais ou d'un langage informatique dérivé de l'anglais, comme le COBOL, est inversement proportionnelle au niveau de connaissances e de compétences : le pupitreur, au contact direct avec des machines et des langages de commande, décalques les messages-écran et les instructions qu'il manipule, le technicien, puis l'ingénieur et enfin le chercheur utilisent des concepts et des notions de plus en plus théoriques et abstraits qu'ils ont tendance à formuler avec des mots moins directement issus des matériels et logiciels qu'ils utilisent. Par ailleurs, de plus en plus en contact avec les utilisateurs, ils doivent être compris.

L'anglais scientifique en général n'en demeure pas moins une langue souvent difficile à comprendre pour le non-scientifique anglophone. Le scientifique préférera par exemple les mots d'origine latine. Pour nos étudiants français, cela rend cette langue plus facile que la langue idiomatique d'origine saxonne. De même, le scientifique privilégiera des tournures de phrases logiques plutôt que chronologiques, inspirées des périodes de la rhétorique classique, et ne reculera pas devant les lourdeurs et les répétitions destinées à lever tout risque d'ambiguïté.

\subsection{La démarche de pédagogue de l'anglais scientifique}

Des scientifiques anglophones essaient de lutter contre cette tendance à l'hermétique d'une classe professionnelle qui cherche plus ou moins inconsciemment à conserver le pouvoir du savoir. Quelques exemples tirés de Scientists must write: A guide to better writing 
for scientists, engineers and students (Barras 1986) et de Effective Writing: Improving scientific, technical and business communication (Turk \& Kirkman 1989) sont très révélateurs. On peut s'interroger sur cette démarche. Certes, des lourdeurs peuvent être supprimées sans perte d'information. Cependant, le discours scientifique a des spécificités propres aux sujets qu'il traite et la formulation choisie spontanément par le scientifique, comme le passif (condamné comme lourd), n'est pas indépendante de la pensée qu'il véhicule. La répétition est peut-être redondante pour un littéraire, elle est utile pour le scientifique qui ne peut risquer l'ambiguïté et pour qui la fréquence d'un mot est un indice d'importance du concept exprimé. La préférence de la langue scientifique pour la nominalisation aux dépens des formes verbales plus caractéristiques du discours anglosaxon est aussi la marque d'une conceptualisation des phénomènes analysés. Alors que la description est volontiers verbale, l'énoncé d'une règle ou d'un théorème isole le fait scientifique dans un terme qui exprime et résume le concept étudié ou découvert.

\subsection{Deux exemples extrêmes}

D'une part, un catalogue de logiciels de Control Data (voir annexe 3) et, d'autre part, le manuel universitaire humoristique d'enseignement du FORTRAN (Kaufman 1979). La langue professionnelle directe et sans fioritures est accessible pour l'apprenant. L'humour et les tournures idiomatiques constituent un filtre et un écran à la compréhension : l'orchestration « littéraire » éloigne le lecteur de l'objet de son étude et exige de lui une culture et une compétence linguistique beaucoup plus importante, qu'il n'est pas toujours possible de lui dispenser dans le temps imparti pour l'étude de la langue anglaise.

\subsection{Une preuve}

Le projet de fin d'études d'anglais dans notre département d'IUT comporte un dossier constitué à partie d'articles sélectionnés dans des revues professionnelles comme ComputerWorld, Byte, Personal Computer World (cf. Dechet 1991). Les étudiants doivent surligner les mots qui leur posent problème ou qu'ils estiment devoir poser problème à un étudiant de leur niveau et ils doivent en fournir une traduction en français.

Dans son dossier sur "Data security " (la protection des données), la meilleure étudiante d'anglais de la promotion s'est trouvée confrontée à deux colonnes : les forces du bien, les informaticiens, les spécialistes, ceux qui protègent les données, «the Protectors» et les forces du mal, les pirates, «the Invaders ». Pour la première catégorie, aucun problème de vocabulaire, pour les seconds, «to jimmy», «snooks» et «stick-to-itveness» avaient (on pouvait s'y attendre) posé problème. Cette dichotomie très nette dans le choix des termes et même dans le registre du discours est tout à fait révélatrice et symptomatique. Entre informaticiens, on parle une langue certes un peu codée et sans grande fantaisie, mais de ce fait facilement intelligible. L'outsider a droit à l'argot et aux néologismes.

De même, dans des textes de la presse professionnelle non informatique comme Fortune et surtout dans les rubriques de vulgarisation des grands magazines Newsweek et Time, ce ne sont pas les termes techniques (généralement connus en fin de formation), mais les termes journalistiques ou les métaphores qui ont posé problème. Ainsi la revue de vulgarisation se révèle plus riche d'enseignement à dispenser pour l'enseignant de 
langue, mais c'est aussi une sorte de luxe quand la durée de la formation est limitée dans le temps.

\section{Conclusion}

31 Les pistes de recherche ici tracées pour l'anglais informatique peuvent être suivies par les autres spécialités. Si le niveau de compétence professionnelle de l'apprenant détermine la nature de l'enseignement à lui dispenser, il conditionne aussi grandement le niveau de compétence de l'enseignant dans la spécialité de l'apprenant. Pour un ingénieur en informatique de haut niveau, l'enseignant peut ne pas posséder de grandes compétences en informatique; une culture générale dans la discipline et la profession lui suffit. Pour un futur DUTien, qui passe en cinq trimestres d'un niveau zéro en informatique à un niveau bac +2 , l'enseignant de langue doit faire l'effort de l'accompagner et de le suivre dans son parcours vers la professionnalisation.

L'enseignant doit donc s'intégrer à l'équipe pluridisciplinaire du département pour s'adapter aux nécessités linguistiques de l'apprenant dans sa discipline. Il doit toutefois veiller à ne pas anticiper sur le cours de spécialité en choisissant des thèmes non encore étudiés. Il doit savoir suivre chez l'apprenant «le progrès scientifique et le développement de la langue" (Gallais-Hamonno 1982b: 40). Il doit être capable de repérer quand l'utilisateur de l'effaceur d'une machine à écrire l'utilise sans plus même y penser, quand un concept de « langage » est devenu un terme du " discours scientifique », voire de la trame rhétorique du « discours pédagogique » de la spécialité.

\section{BIBLIOGRAPHIE}

Barras, Robert. 1986 [1978]. Scientists must write: A guide to better writing for scientists, engineers and students. Londres, New York : Chapman and Hall.

Dechet, Arlette. 1991. «Le projet de fin de formation en anglais de l'informatique. Les Cahiers de 1'APLIUT 42-43, 41-55.

Gallais-Hamonno, Janine. 1979. The Language of Basic Economics, manuel d'anglais économique. Paris : Dunod.

Gallais-Hamonno, Janine. 1980. « Les emprunts réciproques de la langue spécialisée et de la langue commune ». Cahiers de l'institut de linguistique de Louvain 6, /1-2, 181-191.

Gallais-Hamonno, Janine. 1982a. Langage, langue et discours économique. Paris : Klinsieck (Thèse de doctorat d'état, Université Paris 3, 1978).

Gallais-Hamonno, Janine. 1982b. « Niveaux d'énonciation, niveaux de culture ». Les Cahiers de 1'APLIUT 5, 28-46.

Hanks, Patrick. 1988. « A new kind of dictionary for English learners: Cobuild ». Les Cahiers de l'APLIUT 30-31 (8/1-2), 73-88. 
Hanks, Patrick. 1989. « How common is common ». In Collins Dictionary Diary, 2-12.

Hockey, Susan. 1992. "Computerised text analysis: The Oxford text searching system ». In Actes $\mathrm{du} 10^{\mathrm{e}}$ colloque du GERAS. Toulouse : Civilisations 4, 23-48.

Kaufman, Roger Emanuel. 1979. A Fortran Coloring Book: Learn computer programming the painfully funny way. Boston : The MIT Press.

McDonough, Jo. 1984. ESP in Perspective: A practical guide. Londres et Glasgow : Collins ELT.

Leray, Yvette, Anne Pradeilles et Nicole Vigouroux-Frey. 1980. This Quiet Revolution. Paris : Longman-Nathan.

Rivas, Michèle. 1988. « Dictionnaire anglais unilingue et autonomie de l'apprentissage, de l'utilité du Collins Cobuild English Language Dictionary en milieu universitaire ». Les Cahiers de l'APLIUT 30-31 (8/1-2), 89-104.

Robinson, Pauline. ESP: The current position. Londres : Pergamon Press.

Roe, Peter. 1977. « Scientific Text: Selections from the linguistic evidence presented in a study of difficulty ». Discourse Analysis Monographs 4. Birmingham : English Language Research, Birmingham University.

Roe, Peter. 1981. « A task-based approach to ESP ». Les Cahiers de l'APLIUT 2, 101-108.

Tournier, Jean. 1988. Précis de lexicologie. Paris : Nathan.

Trimble, Louis. 1985. English for Science and Technology: A discourse approach. Cambridge :

Cambridge University Press.

Turk, Christopher \& John Kirkman. 1989 [1982]. Effective Writing: Improving scientific, technical and business communication. Londres, New York : E \& F.S. Spon.

Actes de colloques et revues pédagogiques à caractère scientifique

Actes des colloques du GERAS Bordeaux 2, 1979 et 1981 ; Montpellier 3, 1982 ; Paris 9 Dauphine 1986 ; IUT Paris 5, 1988 ; Toulouse 11989 ; Bordeaux 2, 1990.

Les Cahiers de l'APLIUT 2, 3, 4, 5, 6 et suivants.

Ateliers d'anglais de spécialité des congrès de la SAES, dont quelques communications dans les Cahiers de l'APLIUT et depuis 1989, regroupées avec les actes des colloques du GERAS.

Les Cahiers de l'ILSER, Université Montpellier 3, Institut de langue de spécialité : Enseignement et recherche numéros 1 (jan. 1983 ; 2 (nov. 1983) ; 3 (mai 1984) ; 5 (jan 1985). En particulier, articles de J.-M. Baïssus ; T. Lattes ; J. Martin ; T. Toma et J.-L. Trouillon.

Publications du GEPE (Groupe d'étude pour le plurilinguisme européen), Université de Strasbourg, notamment

Le Guilly-Wallis, Anne. 1984. « Le franglais : nature et cause. Étude de cas. Le franglais dans

l'informatique. Actes du $1^{\text {er }}$ colloque du GEPE « L'anglais : langue étrangère ou langue seconde », mai 1984.

Prince, Violaine. 1986. «La pidginisation du français par le jargon américain de l'informatique ». Actes du $2^{e}$ colloque du GEPE « Langue française - langue anglaise: contacts et conflits », mai 1986.

\section{Études de l'auteur}

Pradeilles, Anne. 1978. «L'enseignement de l'anglais aux étudiants des départements

Informatique des IUT. In Actes du $18^{\mathrm{e}}$ congrès de la SAES, Limoges 1978, Études anglaises 78, 53-58. 
Pradeilles, Anne. 1981a. «L'interdisciplinarité, une formation continue indispensable pour l'enseignant de langue de spécialité ». In Actes du $4^{e}$ colloque du GERAS. Bordeaux : Université Bordeaux 2, 101-113.

Pradeilles, Anne. 1981b. «L'analyse du 'discours pédagogique' comme technique de lecture rapide et d'aide au résumé ». Communication au $22^{\mathrm{e}}$ congrès de la SAES, Université Lyon 2 mai 1981.

Pradeilles, Anne. 1981c. «A new C.A.R. for E.S.P. teachers : quelques modestes propositions pour la recherche assistée par ordinateur en anglais de spécialité ». Les Cahiers de l'APLIUT 2, 91-100.

Pradeilles, Anne. 1981d. «L'article défini en anglais informatique : analyse quantitative de 'the' de 'langage', 'discours scientifique' et 'discours pédagogique'«. Sigma 6, CELAM Université Valéry, Montpellier, 85-97.

Pradeilles, Anne. 1981e. « Les quatre niveaux d'un énoncé scientifique ou technique ». Les Cahiers de l'APLIUT 3, 50-71.

Pradeilles, Anne. 1982. « Applications pédagogique d'une analyse syntaxique de l'anglais informatique ». Les langues modernes 1, 50-61.

Pradeilles, Anne. 1984. «Une méthodologie du résumé en anglais de spécialité ». Les Cahiers de l'IUT : économie et sciences humaines 7, Université Paris 5 René Descartes, 301-310.

Pradeilles, Anne. 1987. «Pour une informatisation de l'analyse syntaxique des formes verbales en anglais scientifique et technique. Actes du congrès d'Amiens 1982, Études anglaises 94, 440-457.

Pradeilles, Anne, Alison Piper et Arlette . 1987. «L'utilisation du traitement de texte comme outil pédagogique pour améliorer l'expression écrite ». Les Cahiers de l'APLIUT 26, 78-90.

Pradeilles, Anne. 1988. «Élaboration d'un dictionnaire d'anglais des réseaux en TURBO C sur compatible IBM PC et l'analyse qualitative et quantitative d'un corpus de textes anglais sur les réseaux ». Les Cahiers de l'APLIUT 30-31, 105-110.

Pradeilles, Anne. 1992. « Le voir et le dire : nouvelles perspectives offertes par le scanner en linguistique appliquée et didactique des langues ». In Annales de Savoie 15, 165-184.

\section{ANNEXES}

Annexe 1 : Schémas tirés de P. Robinson (1980) et P. Strevens (1981) 


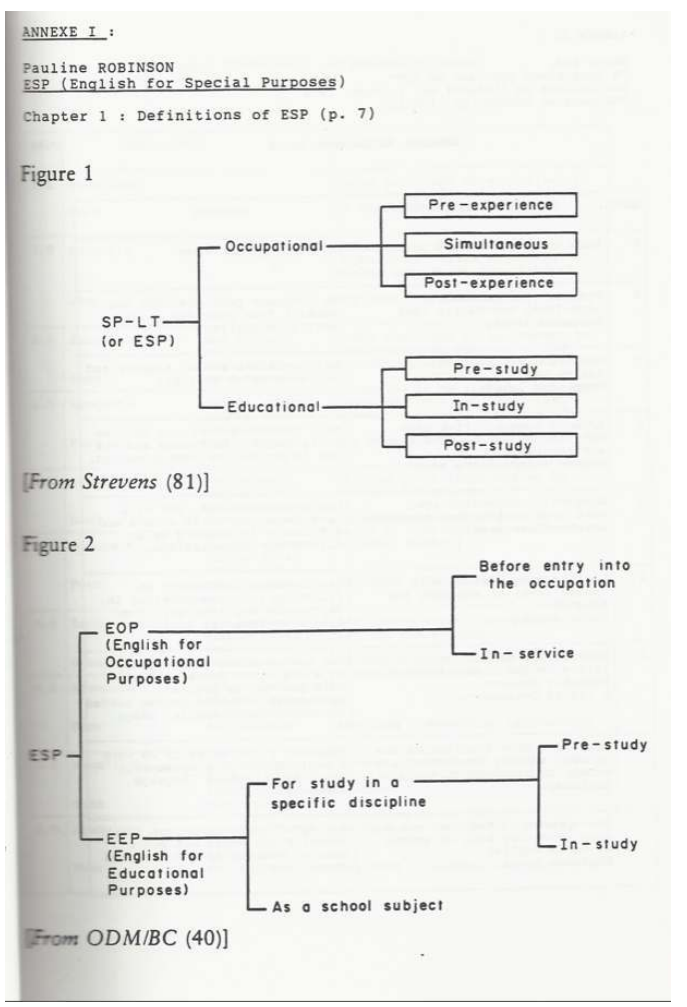

Annexe 2 : Schémas

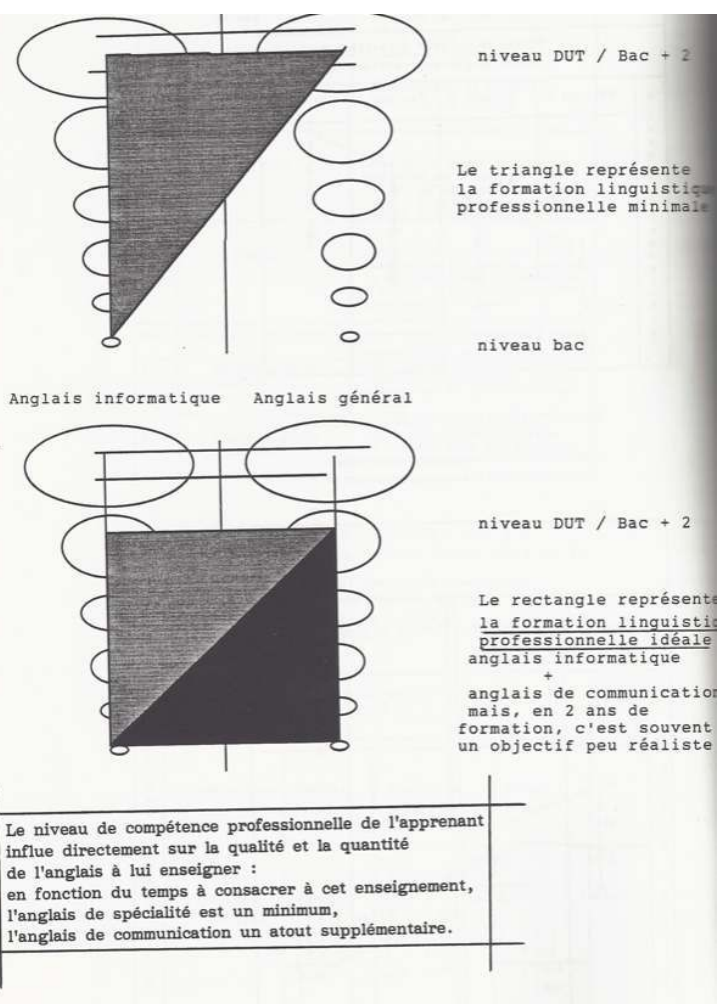

Annexe 3 : Extrait d'un catalogue de logiciels du constructeur américain d'ordinateurs, Control Data. L'exemple du FORTRAN et du PSS/E (Power System Simulator for Engineering) 


\begin{tabular}{|c|c|}
\hline TITRAN & \\
\hline Eileation & Compilers/Utilities \\
\hline Zneription & $\begin{array}{l}\text { Compiler for the FORTRAN programming language. } \\
\text { Includes math and input/output libraries. Implements } \\
\text { ANSI FORTRAN } 77 \text { (X3.9-1978) language plus } \\
\text { extensions. }\end{array}$ \\
\hline $\begin{array}{l}\text { Gerating } \\
\text { Aystem }\end{array}$ & NOS/VE, NOS, NOS/BE \\
\hline Decumentation & Reference/Usage Manual \\
\hline 3upport & Telephone, Mail-In, Standard Contract \\
\hline Deographic Area & Worldwide \\
\hline installation & User \\
\hline Training & Not Required; 5-day workshop available \\
\hline $\begin{array}{l}\text { Education Use } \\
\text { Discount } \\
\text { Available }\end{array}$ & Yes \\
\hline Comments & Standard product \\
\hline Contact & Control Data \\
\hline
\end{tabular}

\section{AUTEUR}

ANNE PRADEILLES 